\title{
CD44 Expression in Dysplastic and Non-Dysplastic Oral Lichen Planus
}

\author{
Narges Ghazi (iD) ${ }^{1}$, Nasrollah Saghravanian ${ }^{1}$, Ala Ghazi (ib) ${ }^{2,}$, , Mohammad Taghi Shakeri ${ }^{3}$ and \\ Hamidreza Khajehbahrami ${ }^{4}$ \\ ${ }^{1}$ Department of Oral and Maxillofacial Pathology, School of Dentistry, Mashhad University of Medical Sciences, Mashhad, Iran \\ ${ }^{2}$ Oral and Maxillofacial Diseases Research Center, Mashhad University of Medical Sciences, Mashhad, Iran \\ ${ }^{3}$ Department of Community Medicine and Public Health, Mashhad University of Medical Sciences, Mashhad, Iran \\ ${ }^{4}$ School of Dentistry, Mashhad University of Medical Sciences, Mashhad, Iran \\ "Corresponding author: Oral and Maxillofacial Diseases Research Center, Mashhad University of Medical Sciences, Mashhad, Iran. Email: alaghazi10@gmail.com
}

Received 2019 September 13; Accepted 2019 November 13.

\begin{abstract}
Background: Oral lichen planus (OLP) is a relatively common chronic inflammatory autoimmune disease. OLP is more resistant than skin form and malignant transformation into oral squamous cell carcinoma (OSCC) has been reported in this lesion. Since SCC often originates from dysplastic lesions, evaluation of cancer stem cell (CSC) markers in dysplastic and premalignant lesions such as OLP seems to be necessary. Furthermore, chronic inflammation may induce some epithelial malignancies. CD44 as a CSC marker is expressed on epithelial cells and contributes to cell migration and tumor progression. Also, this cell adhesion molecule is expressed on leukocytes involved in various inflammatory diseases.

Methods: Thirty samples of erosive/atrophic OLP (15 samples of dysplastic OLP and 15 samples of non-dysplastic OLP), and 10 normal mucosa specimens of the oral cavity were examined using immunohistochemistry.

Results: The greatest total score for epithelial immunoreactivity was found in dysplastic OLP, followed by non-dysplastic OLP and normal mucosa with significant differences $(\mathrm{P}<0.001)$. For sub-epithelial lymphocytes immunoreactivity, the greatest total score was reported in dysplastic OLP, followed by non-dysplastic OLP.

Conclusions: Significant higher expression of CD44 as a CSC marker in the epithelium of dysplastic OLP may show the role of this marker in malignant transformation and carcinogenesis process of this premalignant lesion. Higher immunoreactivity in subepithelial lymphocytes of dysplastic type indicates a probable role of chronic inflammation in the malignant potential of OLP.
\end{abstract}

Keywords: Lichen Planus, Dysplastic, CD44

\section{Background}

Oral lichen planus (OLP) is a relatively common chronic inflammatory autoimmune disease that activates cytotoxic $\mathrm{T}$ lymphocytes against epithelial cells. OLP is more resistant than skin form and malignant transformation into oral squamous cell carcinoma (OSCC) has been reported in this lesion (1). A large number of OSCCs in the early stages clinically appear as premalignant lesions or potentially malignant oral lesions (PMOLs) (2). Since the risk of malignant transformation to OSCC for OLP lesions has been reported, World Health Organization (WHO) categorizes these lesions as PMOLs $(3,4)$. Therefore, early diagnosis of PMOLs including OLP is of critical importance. Therefore, using biomarkers to evaluate the disease at the early stages leads to the improvement of preventive therapies to suppress the disease in the initial phase (1).

It has been reported that the development of cancerous tumors is exclusively dependent on the functional role of cancer stem cells (CSCs) that cause the emergence of a new model of carcinogenesis known as cancer stem cells hypothesis. Since OSCC often originates from dysplastic lesions, evaluation of CSC markers in dysplastic and premalignant lesions seems to be necessary $(1,5,6)$.

Among the markers of cancer stem cells, CD44 can be mentioned as a cell surface glycoprotein. The expression of CD44 is a distinct feature of the cancer-initiating cells and contributes to tumor endothelial reactions, cell migration, cell adhesion, tumor invasion, prognosis, and metastasis $(7,8)$. Since CD44 is expressed on epithelial cells, we evaluated this CSC marker in the epithelium of non-dysplastic OLP and dysplastic type as a PMOL lesion.

Recent molecular studies have given a new perspective on the association between malignant tumors and surrounding stroma. There is now enough evidence to accept the fact that the chronic inflammatory process is capable of creating a cytokine-based microenvironment. This growth environment can induce proliferation, differentia- 
tion, and movement of the cells. It also contributes to progression, invasion, and metastasis of cancer (9).

\section{Objectives}

CD44, as a cell adhesion molecule, is expressed on epithelial cells as well as on leukocytes, and along with its ligand hyaluronan (HA), is involved in various inflammatory diseases including OLP. Investigation of the role and functional mechanism of this marker can serve as a therapeutic goal in chronic inflammatory diseases (10). Therefore, in the present study, in addition to evaluation of CD44 in OLP epithelium, the expression of this marker in sub-epithelial lymphocytes was also investigated.

\section{Methods}

In this study, 40 samples including 15 samples of dysplastic (moderate dysplasia) lichen planus, 15 samples of non-dysplastic lichen planus, and 10 normal mucosa samples of the oral cavity were examined using immunohistochemistry.

This study included individuals with erosive/atrophic OLP referred to the Department of Oral Medicine, School of Dentistry, Mashhad University of Medical Sciences. The cases with fulfillment both clinical and histopathological features consistent with the World Health Organization (WHO) criteria were selected. The selection criteria included:

1. bilateral presence of papules or reticulate lesions, with or without atrophic/erosive lesions.

2. Bandlike zone of lymphocytes infiltration with signs of degenerative liquefaction of the basal cell layer (11).

Tissues were sectioned and evaluated histologically after staining in hematoxylin and eosin. Routine histological examination was independently reconfirmed by 2 experienced professionals. After confirmation of the cases, the hematoxylin- and eosin-stained sections were studied under low power $(10 \times)$ and high power $(40 \times)$ magnifications for the valuation of the dysplastic features.

Immunohistochemistry (IHC) was applied on $4 \mathrm{um}$ formalin-fixed and paraffin-embedded tissue sections. Tissue sections were deparaffinized in xylene and rehydrated in decreasing ethanol series. In order to antigen retrieval, the sections went through the 0.01 citrate solution $(\mathrm{pH}=$ 6.0) for $10 \mathrm{~min}$. To block endogenous peroxidase activity, solution of methanol with $0.5 \%$ hydrogen peroxide was used for $10 \mathrm{~min}$. Tissue sections were then washed in Trisbuffered saline (TBS), $\mathrm{pH}=7.6$ and incubated with diluted normal serum for $10 \mathrm{~min}$. The following monoclonal antibody was applied for $30 \mathrm{~min}$ at room temperature: anti-
CD44 (clone DF1485, 1:50 dilution, Novocastra Laboratories, Newcastle, UK).

The immunostains were reviewed by two pathologists. The slides were examined with a light microscope at a final magnification of $400 \times$. Positivity was considered according to the percentage of positive epithelial cells and sub-epithelial lymphocytes. Immunohistochemical reactivity for CD44 (designated as labeling index, LI) was scored as follows: CD44 immunoreactivity :4+, more than $75 \%$; $3+$, $50 \%-75 \% ; 2+, 25 \%-50 \% ; 1+, 5 \%-25 \%$; and negative (-), less than $5 \%(4,12)$.

All analyses were performed using data processing program SPSS/PC version 22.0 for Windows. Kruskal-Wallis and Dunn-Bonferroni post hoc test were used to assess the statistically significant differences between studied groups. Statistical significance was defined as $\mathrm{P}<0.05$.

\section{Results}

Of 30 patients with OLP, 12 were males and 18 were females with a mean age of $12.36 \pm 46.73$ years. There was no significant difference between the two groups regarding gender and age.

CD44 was expressed in all lining epithelial cells and sub-epithelial lymphocytes of OLPs with different degrees while $30 \%$ of normal mucosa samples showed negative epithelial immunoreactivity. Representative staining is shown in Figures 1-3, and the entire dataset are reported in Tables 1 and 2.

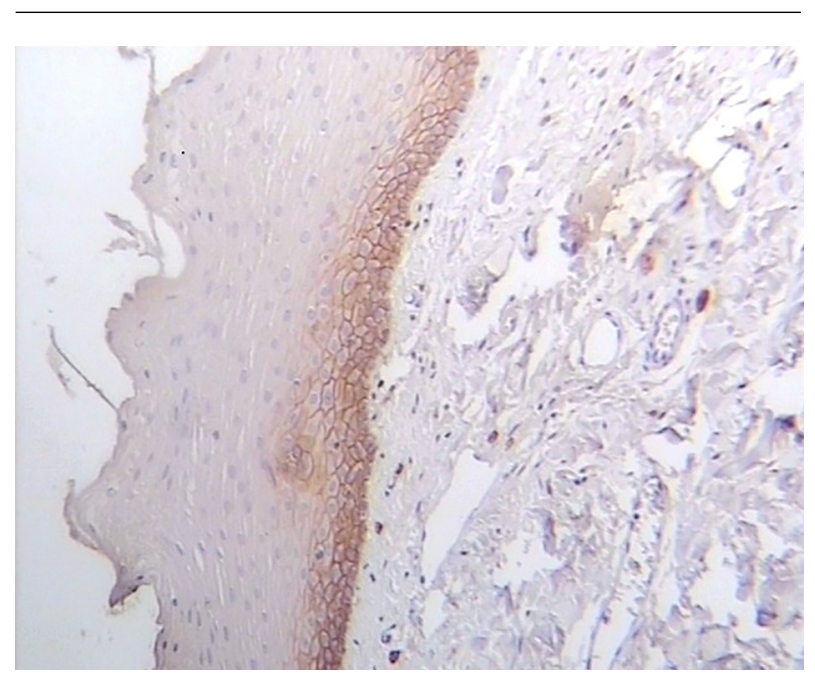

Figure 1. CD44 expression in the epithelium of normal mucosa

In most dysplastic OLP cases, CD44 labeling index in the epithelium and sub-epithelial lymphocytes was strong 


\begin{tabular}{|c|c|c|c|c|c|c|c|c|c|}
\hline \multirow{2}{*}{ Lesion } & \multicolumn{7}{|c|}{ CD44 LI, No. (\%) } & \multirow{2}{*}{ Mean Total Score } & \multirow{2}{*}{ Standard Deviation (SD } \\
\hline & \multicolumn{3}{|c|}{$1+$} & \multicolumn{2}{|c|}{$2+$} & $3+$ & $4+$ & & \\
\hline $\operatorname{Normal}$ mucosa $(n=10)$ & $3(30)$ & \multicolumn{2}{|c|}{$7(70)$} & \multicolumn{2}{|c|}{0} & 0 & 0 & 0.70 & 0.48 \\
\hline Non-dysplastic OLP $(n=15)$ & 0 & \multicolumn{2}{|c|}{0} & \multicolumn{2}{|c|}{$6(40)$} & $7(46.7)$ & $2(13.3)$ & 2.74 & 0.70 \\
\hline Dysplastic OLP $(n=15)$ & 0 & \multicolumn{2}{|c|}{0} & \multicolumn{2}{|c|}{$1(6.7)$} & $2(13.3)$ & $12(80)$ & 3.74 & 0.59 \\
\hline \multirow{2}{*}{ Lesion } & \multicolumn{7}{|c|}{ CD44 LI, No. (\%) } & \multirow{2}{*}{ Mean Total Score } & \multirow{2}{*}{ Standard Deviation (SD) } \\
\hline & $\mathbf{0}$ & $\mathbf{1 +}$ & & & & & + & & \\
\hline Non-dysplastic OLP $(n=15)$ & 0 & 0 & & & & 3.3) & 33.3) & 3.20 & 0.67 \\
\hline Dysplastic OLP $(n=15)$ & 0 & 0 & & & & 20) & 53.3) & 3.66 & 0.61 \\
\hline
\end{tabular}

(4+) (Figure 2). The greatest total score for epithelial immunoreactivity was found in dysplastic OLP, followed by non-dysplastic OLP and normal mucosa (Table 1). A comparison of the total score among the three groups revealed significant differences $(\mathrm{P}<0.001)$. Also, the Pairwise comparison of CD44 expression in the studied groups was performed. According to Dunn-Bonferroni post hoc test all the paired groups demonstrated significant differences ( $P$ $<0.01$ ).

Sub-epithelial lymphocytes immunoreactivity was evaluated in dysplastic and non-dysplastic OLP groups. The greatest total score was reported in dysplastic OLP, followed by non-dysplastic OLP (Table 2). According to Mann-Whitney test, there was no statistically significant difference between the two groups $(\mathrm{P}=0.067)$.

\section{Discussion}

OLP is a relatively common chronic inflammatory autoimmune disease. OSCC can be preceded by potentially malignant lesions such as OLP (4). Although the WHO classifies OLP as a potentially malignant oral lesion, there is considerable controversy regarding the malignant potential of this lesion. This controversy is due to a wide range of malignant transformation rates (0\% - $12.5 \%)$ in OLP patients as well as different clinical criteria used for diagnosis of this lesion. Nevertheless, most studies demonstrated that OLP patients develop oral cancer at an increased rate (approximately 10 times higher risk) when compared to the general population $(13,14)$. A meta-analysis study also reported malignant transformation of OLP six years after the initial diagnosis with the mean rate of $0.27 \%$ per year (14).

Lodi et al. reported that the presence of epithelial dysplasia in an OLP lesion may correspond to two types of conditions: lesions with clinical features of OLP but with dysplasia at histological level and lesions with lichenoid histopathological features (particularly band-like inflammatory infiltration) but without the classic clinical features of OLP (e.g. with unilateral distribution or absence of reticular lesions). The former may represent an early phase of OLP malignant transformation, whereas the latter may correspond to one of the various clinical conditions (15). In addition, it is probably well accepted that the final diagnosis of OLP cannot be made on histopathological features alone and that often clinical and histopathological evaluation is required (16). Therefore, in order to achieve appropriate results in the present study, cases with fulfillment both clinical and histopathological features of OLP were selected. Concerning the clinical form of OLP, numerous authors have found that atrophic-erosive forms predisposed to cancer development (15).

The role of CSCs in epithelial dysplasia, tumor development, growth, and metastasis has been approved and investigated by various studies. Malignant transformation of dysplastic epithelium is reported to be $3 \%$ to $36 \%$ (1). Therefore, evaluation of CSC markers, including CD44, seems to be necessary for dysplastic and premalignant lesions such as OLP.

It has been shown that chronic inflammation may induce some epithelial malignancies. The relation between chronic esophagitis and esophageal adenocarcinoma as well as intestinal cancer and chronic inflammatory bowel disease has been reported (9).

It should be noted that inflammation itself, the basic pathological feature of OLP, can elicit histological features similar to mild dysplasia (15). Moreover, particularly in mild cases of dysplasia chronic inflammatory host response to the atypical cells of epithelial dysplasia can appear histopathologically similar to OLP (3). Therefore, although most of our reporting system included a diagnostic category of mild and moderate dysplastic OLP, we evaluated only moderate dysplastic OLP in our study. 

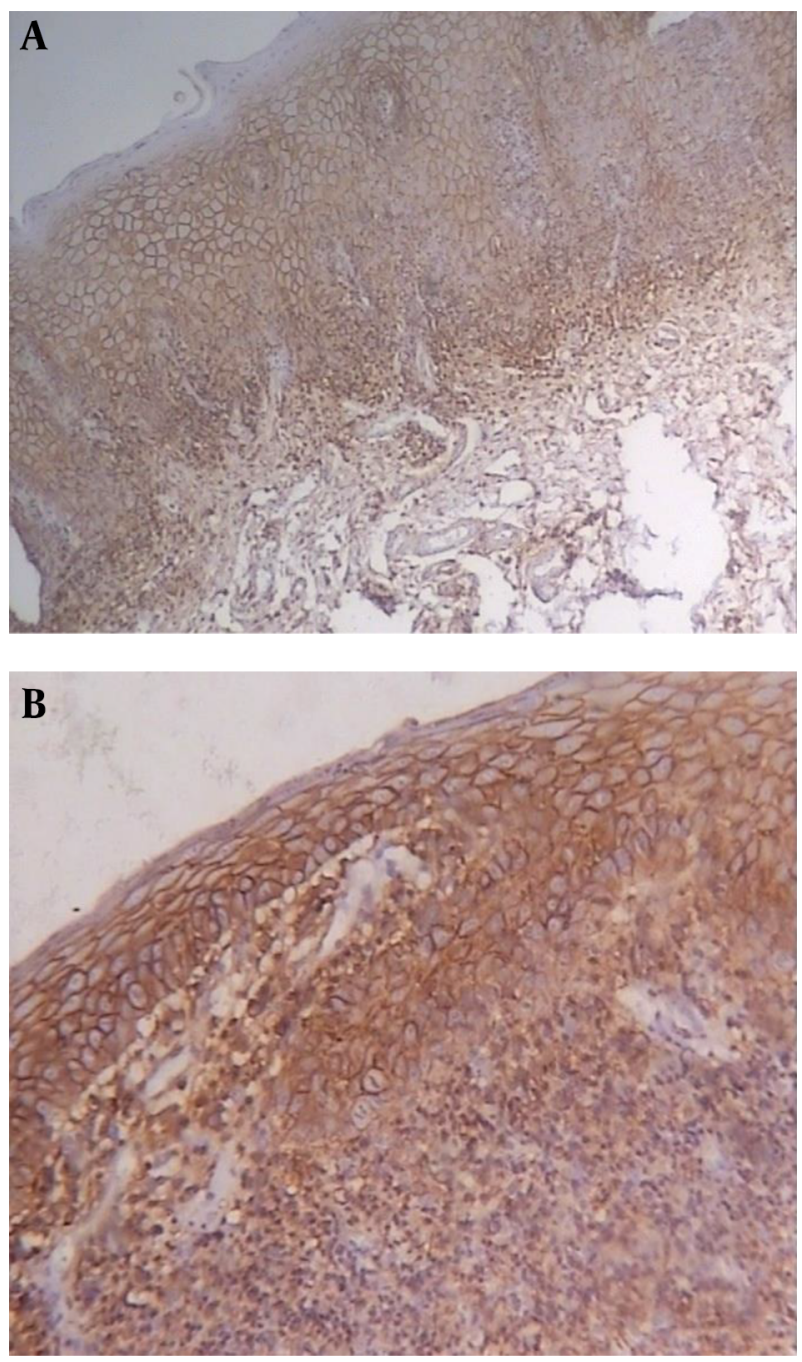

Figure 2. Dysplastic OLP showing immunostaining (4+) in the epithelium and subepithelial lymphocytes $(\mathrm{A}, 400 \times)$ and $(\mathrm{B}, 200 \times)$

CD44, as a cell adhesion molecule is expressed on epithelial cells and also leukocytes. This marker along with its ligand, hyaluronan (HA), is involved in various inflammatory diseases, including OLP. Investigating the role and functional mechanism of CD44 can serve as a therapeutic goal in chronic inflammatory diseases (10).

In the present study, CD44 expression as a CSC marker was evaluated in dysplastic and non-dysplastic OLP. Furthermore, the expression of this marker in sub-epithelial lymphocytes of OLP as a chronic inflammatory disease was investigated. Some other studies (17) evaluated definitely epithelial dysplasia, whereas in the current study, after confirming the OLP diagnosis, the dysplastic changes in OLP lesions were evaluated. Therefore, to the best of our
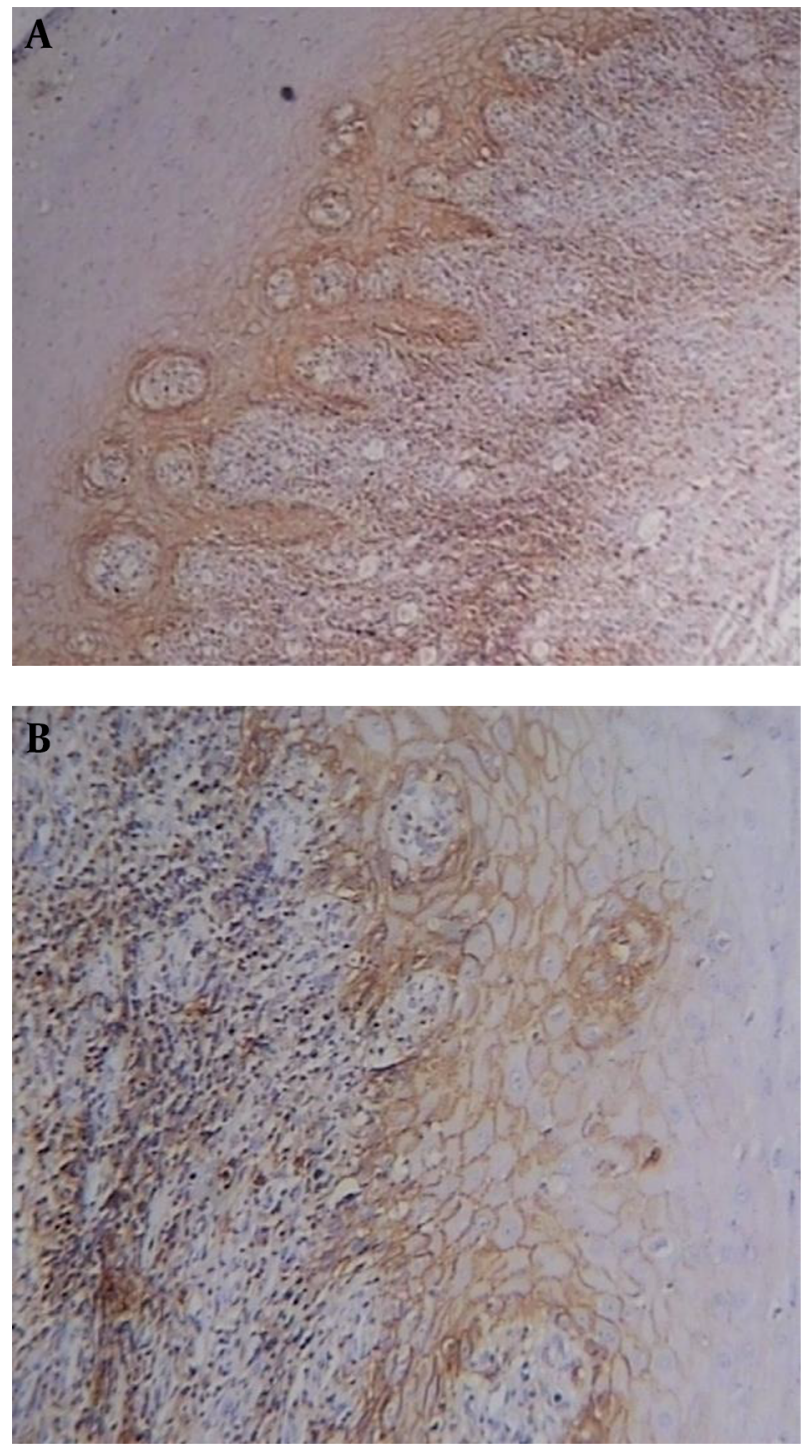

Figure 3. Non-dysplastic OLP $(\mathrm{A}, 100 \times)$ : immunostaining in the epithelium $(1+)$ and subepithelial lymphocytes $(2+),(B, 400 \times)$ : immunostaining in the epithelium $(2+)$ and subepithelial lymphocytes $(4+)$

knowledge, CD44 expression in dysplastic OLP was evaluated for the first time in this study.

According to our results, higher expression of CD44 was reported in dysplastic lesions which shows the role of this marker in the carcinogenesis process as a CSC marker. Consistent with the findings of the present study, Kaya et al. also showed a high CD44 expression in epithelial keratinocytes of OLP (18).

Santarelli et al. reported that CD44 and osteopontin expression in epithelium of OLP patients were significantly higher than the control group and they concluded that 
these markers could play a role in the pathogenesis of OLP (19). Siponen et al. also demonstrated a higher expression of hyaluronan, CD44, HASI-3, and HYALI-2 in the epithelium of OLP patients compared to the control group (20).

Since CD44 is expressed on both lymphocytes and parenchymal cells, in addition to the role of this marker in the carcinogenesis process, its role in autoimmune diseases has also been taken into account in recent years (10).

One of the early events in inflammatory responses is the recruitment of inflammatory cells that are mediated by cell-adhesion molecules such as CD44 and the production of chemical adsorbents. Evidence showed that CD44 plays a major role in various inflammatory diseases and according to the previous studies the application of the Anti-CD44 antibody can control inflammatory bowel disease and skin encephalomyelitis (10).

In the current study, the expression of CD44 in OLP samples demonstrates its role in the recruitment of $\mathrm{T}$ lymphocytes in the pathogenesis of this lesion. In the study by Liu et al. most of the T cells infiltrated in the OLP lamina properia showed high CD44 immunoreactivity (21).

Mignogna et al. also evaluated the role of inflammation in the malignant transformation of OLP. According to their study, sub-epithelial infiltration of inflammatory cells and cytokines network can be associated with the tumorigenesis and malignant transformation of OLP (9).

Yasuda et al. also reviewed the relevance of CD44 with inflammation and malignancy. They stated that CD44 as a major receptor for hyaluronan (an essential component of the extracellular matrix) has an important role in the inflammation process and potential for tumor formation. Therefore, a special focus should be placed on CD44mediated signaling pathways in therapeutic approaches (22).

Similar to our results, Kaya et al. investigated CD44 isoforms including (CD44s and CD44V3-10) in OLP and observed CD44s immunoexpression in sub-epithelial lymphocytes and epithelial keratinocytes. Epithelial immunoreactivity of CD44v isoforms was decreased or absent. They concluded that the expression of CD44 isoforms in OLP is different from cutaneous LP which revealed that CD44 may play a role in the formation of LP in different locations (18).

In the present study, although there was no significant difference for expression of CD44 in sub-epithelial lymphocytes between dysplastic and non-dysplastic lesions, a higher immunoexpression in dysplastic lesions was observed, that indicates the role of this marker in the process of inflammation as well as malignant transformation. As previously noted, epithelial immunoreactivity was significantly higher in dysplastic lesions, which possibly shows the more important role of this marker in the carcinogen- esis process.

\subsection{Conclusions}

Immunoexpression of CD44 in the epithelium and sub-epithelial lymphocytes of OLP indicates the probable role of this marker in the pathogenesis of this lesion as an inflammatory chronic autoimmune disease.

Significant higher expression of CD44 as a CSC marker in the epithelium of dysplastic OLP may show the role of this marker in malignant transformation and carcinogenesis process of this premalignant lesion. Higher immunoreactivity in sub-epithelial lymphocytes of dysplastic type indicates a probable role of chronic inflammation in the malignant potential of OLP similar to some other chronic inflammatory diseases.

\section{Acknowledgments}

None declared.

\section{Footnotes}

Authors' Contribution: Narges Ghazi, Nasrollah Saghravanian, Ala Ghazi, Mohammad Taghi Shakeri, and Hamidreza Khajehbahrami contributed to the design the research, to the analysis of the results and to the writing of the manuscript.

Conflict of Interests: The authors declare no conflict of interest in this study.

Ethical Approval: The study protocol was approved by the Ethics Committee of Mashhad University of Medical Sciences.

Funding/Support: This study was supported by a grant from the Vice Chancellor of Mashhad University of Medical Sciences, Iran.

Informed Consent: The methodology was described to all patients and the written informed consent was obtained from the patients before participating in the study.

\section{References}

1. Dalley AJ, AbdulMajeed AA, Upton Z, Farah CS. Organotypic culture of normal, dysplastic and squamous cell carcinoma-derived oral cell lines reveals loss of spatial regulation of CD44 and p75 NTR in malignancy. J Oral Pathol Med. 2013;42(1):37-46. doi: 10.1111/j.16000714.2012.01170.x. [PubMed: 22643025].

2. Sudbo J, Reith A. Which putatively pre-malignant oral lesions become oral cancers? Clinical relevance of early targeting of high-risk individuals. J Oral Pathol Med. 2003;32(2):63-70. doi: 10.1034/j.16000714.2003.00054.x. [PubMed: 12542827].

3. Neville B, Damm DD, Allen C, Bouquot J. Oral and maxillofacial pathology. St Louis: Saunders; 2016. p. 453-9. 
4. Salehinejad J, Sharifi N, Amirchaghmaghi M, Ghazi N, Shakeri MT, Ghazi A. Immunohistochemical expression of p16 protein in oral squamous cell carcinoma and lichen planus. Ann Diagn Pathol. 2014;18(4):210-3. doi: 10.1016/j.anndiagpath.2014.03.009. [PubMed: 24850170].

5. Shimada Y, Ishii G, Nagai K, Atsumi N, Fujii S, Yamada A, et al. Expression of podoplanin, CD44, and p63 in squamous cell carcinoma of the lung. Cancer Sci. 2009;100(11):2054-9. doi: 10.1111/j.13497006.2009.01295.x. [PubMed: 19681903].

6. Clarke MF, Dick JE, Dirks PB, Eaves CJ, Jamieson CH, Jones DL, et al. Cancer stem cells-perspectives on current status and future directions: AACR Workshop on cancer stem cells. Cancer Res. 2006;66(19):933944. doi: 10.1158/0008-5472.CAN-06-3126. [PubMed: 16990346].

7. Scully C, Eisen D, Porter S, Rogers R. Dermatology of the lips. Isis Medical Media Ltd; 2000. p. 58-71.

8. Essers J, Theil AF, Baldeyron C, van Cappellen WA, Houtsmuller AB, Kanaar R, et al. Nuclear dynamics of PCNA in DNA replication and repair. Mol Cell Biol. 2005;25(21):9350-9. doi: 10.1128/MCB.25.21.93509359.2005. [PubMed: 16227586]. [PubMed Central: PMC1265825].

9. Mignogna MD, Fedele S, Lo Russo L, Lo Muzio L, Bucci E. Immune activation and chronic inflammation as the cause of malignancy in oral lichen planus: is there any evidence ? Oral Oncol. 2004;40(2):120-30. doi:10.1016/j.oraloncology.2003.08.001. [PubMed:14693234].

10. Pure E, Cuff CA. A crucial role for CD44 in inflammation. Trends Mol Med. 2001;7(5):213-21. doi: 10.1016/s1471-4914(01)01963-3. [PubMed: 11325633].

11. Kramer IR, Lucas RB, Pindborg JJ, Sobin LH. Definition of leukoplakia and related lesions: An aid to studies on oral precancer. Oral Surg Oral Med Oral Pathol.1978;46(4):518-39. [PubMed: 280847].

12. Chaiyarit $P$, Thongprasom K, Satayut S, Dhanuthai K, Piboonratanakit $\mathrm{P}$, Phothipakdee P, et al. Alteration of the expression of CD44 [corrected] isoforms in oral epithelia and saliva from patients with oral lichen planus. J Clin Immunol. 2008;28(1):26-34. doi: 10.1007/s10875007-9128-5. [PubMed: 17846873].

13. van der Meij EH, Schepman KP, Plonait DR, Axell T, van der Waal I. Interobserver and intraobserver variability in the clinical assessment of oral lichen planus. J Oral Pathol Med. 2002;31(2):95-8. doi: 10.1046/j.0904-2512.2001.00174.x. [PubMed: 11896830].

14. Liu Y, Messadi DV, Wu H, Hu S. Oral lichen planus is a unique disease model for studying chronic inflammation and oral cancer. Med Hy potheses. 2010;75(6):492-4. doi: 10.1016/j.mehy.2010.07.002. [PubMed: 20674185]. [PubMed Central: PMC3181218].

15. Lodi G, Scully C, Carrozzo M, Griffiths M, Sugerman PB, Thongprasom K. Current controversies in oral lichen planus: report of an international consensus meeting. Part 2. Clinical management and malignant transformation. Oral Surg Oral Med Oral Pathol Oral Radiol Endod. 2005;100(2):164-78. doi: 10.1016/j.tripleo.2004.06.076. [PubMed: 16037774].

16. van der Waal I. Oral lichen planus and oral lichenoid lesions; a critical appraisal with emphasis on the diagnostic aspects. Med Oral Patol Oral Cir Bucal. 2009;14(7):E310-4. [PubMed: 19300362].

17. Bahar R, Kunishi M, Kayada Y, Yoshiga K. CD44 variant 6 (CD44v6) expression as a progression marker in benign, premalignant and malignant oral epithelial tissues. Int J Oral Maxillofac Surg. 1997;26(6):443-6. doi: 10.1016/s0901-5027(97)80010-0. [PubMed: 9418147]

18. Kaya G, Kaya H, Augsburger E, Samson J, Saurat JH, Lombardi T. Expression of CD44 and Its Isoforms in Oral Lichen Planus.JCutaneous Pathol. 2008;32(1):97. doi:10.1111/j.0303-6987.2005.320dm.x.

19. Santarelli A, Mascitti M, Rubini C, Bambini F, Zizzi A, Offidani A, et al. Active inflammatory biomarkers in oral lichen planus. Int J Immunopathol Pharmacol. 2015;28(4):562-8. doi: 10.1177/0394632015592101. [PubMed: 26303119].

20. Siponen M, Kullaa A, Nieminen P, Salo T, Pasonen-Seppanen S. Altered expression of hyaluronan, HAS1-2, and HYAL1-2 in oral lichen planus. J Oral Pathol Med. 2015;44(6):401-9. doi: 10.1111/jop.12294. [PubMed: 25421996].

21. Liu GX, Sun JT, Yang MX, Qi XM, Shao QQ, Xie Q, et al. OPN promotes survival of activated $\mathrm{T}$ cells by up-regulating CD44 in patients with oral lichen planus. Clin Immunol. 2011;138(3):291-8. doi: 10.1016/j.clim.2010.12.007. [PubMed: 21215700].

22. Yasuda M, Nakano K, Yasumoto K, Tanaka Y. CD44: functional relevance to inflammation and malignancy. Histol Histopathol. 2002;17(3):945-50. doi: 10.14670/HH-17.945. [PubMed: 12168806]. 